\title{
Admissible subcategories in derived categories of moduli of vector bundles on curves
}

\author{
Pieter Belmans \\ Swarnava Mukhopadhyay
}

July 3, 2018

\begin{abstract}
We show that the Poincaré bundle gives a fully faithful embedding from the derived category of a curve of sufficiently high genus into the derived category of its moduli space of bundles of rank $r$ with fixed determinant of degree 1. Moreover we show that a twist of the embedding, together with 2 exceptional line bundles, gives the start of a semi-orthogonal decomposition. This generalises results of Narasimhan and Fonarev-Kuznetsov, who embedded the derived category of a single copy of the curve, for rank 2 .
\end{abstract}

\section{Introduction}

Let $C$ be a smooth projective curve of genus $g \geq 2$ and let $r \geq 2$ and $d$ be integers such that $\operatorname{gcd}(r, d)=1$. Then the moduli space $\mathrm{M}_{C}(r, \mathcal{L})$ of stable vector bundles of rank $r$ and fixed determinant $\mathcal{L}$ of degree $d$ is a smooth and projective Fano variety, such that $\operatorname{Pic} \mathrm{M}_{C}(r, \mathcal{L}) \cong \mathbb{Z} \Theta$. Moreover there exists a universal vector bundle $\mathcal{W}$ (called the Poincaré bundle) on $C \times \mathrm{M}_{C}(r, \mathcal{L})$. We can use $\mathcal{W}$ to construct a Fourier-Mukai functor $\Phi_{\mathcal{W}}$ from the derived category of the curve to the derived category of the moduli of vector bundles.

In [14] Narasimhan showed that the Fourier-Mukai functor $\Phi_{\mathcal{W}}$ is fully faithful, if $r=2$, $d=1, g \geq 4$ and $\mathcal{W}$ is suitably normalized (see equation (6)). His proof uses the Hecke correspondence to check the Bondal-Orlov criterion. Independently, in [9] FonarevKuznetsov showed the fully faithfulness for $r=2, d=1$ and $g \geq 2$ when $C$ is a generic curve. Their proof involves an explicit model by Desale-Ramanan [7] of $\mathrm{M}_{C}(2, \mathcal{L})$ when $C$ is hyperelliptic, and checking the Bondal-Orlov criterion in this special case using the Borel-Weil-Bott theorem.

The first result in this article is a generalisation of these fully faithfulness results: the rank $r$ is now arbitrary, the degree $d$ is 1 , and $g \geq g_{0}$, where $g_{0}$ is the smallest integer such that

(1) $\quad 2 r g_{0}-2\left(r+g_{0}\right) \geq r^{2}-1$.

In particular when $r=2$, we get that $g_{0}$ is 4 . The vanishing results in theorem 3 together with the Bondal-Orlov criterion then imply the following. 
Theorem A. The Fourier-Mukai transform $\Phi_{\mathcal{W}}$ gives a fully faithful embedding

(2) $\quad \mathrm{D}^{\mathrm{b}}(C) \rightarrow \mathrm{D}^{\mathrm{b}}\left(\mathrm{M}_{C}(r, \mathcal{L})\right)$

for any smooth curve $C$ of genus $g \geq g_{0}$.

In [14, remark 4] Narasimhan explains that the results in op. cit. also show that

$$
\Theta^{\vee}, \mathcal{O}_{\mathrm{M}_{C}(2, \mathcal{L})}, \Phi_{\mathcal{W}}\left(\mathbf{D}^{\mathrm{b}}(C)\right)
$$

is the start of a semiorthogonal decomposition of $\mathrm{D}^{\mathrm{b}}\left(\mathrm{M}_{C}(r, \mathcal{L})\right)$. The second result in this article is a generalisation and more interestingly an extension of this result to higher rank: we exhibit a second copy of the derived category of the curve. To do this we twist the functor $\Phi_{\mathcal{W}}$ by $\Theta^{\vee}$, or equivalently we consider the Fourier-Mukai functor associated to $\mathcal{W} \otimes p_{2}^{*}\left(\Theta^{\vee}\right)$.

Theorem B. Let $C$ be any smooth curve of genus $g \geq g_{0}$, then there exists a semiorthogonal decomoposition of the form

$$
\mathbf{D}^{\mathrm{b}}\left(\mathrm{M}_{C}(r, \mathcal{L})\right)=\left\langle\Theta^{\vee}, \Phi_{\mathcal{W}}\left(\mathbf{D}^{\mathrm{b}}(C)\right) \otimes \Theta^{\vee}, \mathcal{O}_{\mathrm{M}_{C}(r, \mathcal{L})}, \Phi_{\mathcal{W}}\left(\mathbf{D}^{\mathrm{b}}(C)\right), \mathcal{A}\right\rangle,
$$

where $\mathcal{A}$ is the left-orthogonal complement to the admissible subcategory generated by the 2 exceptional objects and the 2 copies of $\mathrm{D}^{\mathrm{b}}(C)$.

Remark 1. This result is already new in the case of $r=2$. In a work in progress joint with Sergey Galkin [3] we are studying $\mathrm{D}^{\mathrm{b}}\left(\mathrm{M}_{C}(2, \mathcal{L})\right)$ from the point of view of mirror symmetry for Fano varieties:

1. quantum cohomology can be used to give expectations on natural semiorthogonal decompositions;

2. there are various conjectures [10] regarding the eigenvalues of the quantum multiplication $\mathrm{c}_{1}(X) *$ - that can be checked for $\mathrm{M}_{C}(2, \mathcal{L})$.

The suggested decomposition into indecomposable pieces (recall that $\mathrm{D}^{\mathrm{b}}(C)$ is indecomposable by [17]) will involve symmetric powers $\operatorname{Sym}^{i} C$ for $i \leq g-1$. For $r \geq 3$ the picture becomes more complicated, and it is currently unclear what the systematic description could be.

The Bondal-Orlov criterion To check fully faithfulness of the Fourier-Mukai functor $\Phi_{\mathcal{W}}$, we will use following criterion due to Bondal-Orlov [5, theorem 1.1]. We denote the skyscraper at a point $x$ by $k(x)$.

Proposition 2 (Bondal-Orlov). Let $X$ and $Y$ be smooth projective varieties. Let $\mathcal{E}$ be an object in $\mathbf{D}^{\mathrm{b}}(X \times Y)$. Then $\Phi_{\mathcal{E}}$ is fully faithful if and only if

1. $\operatorname{Hom}_{Y}\left(\Phi_{\mathcal{E}}(k(x)), \Phi_{\mathcal{E}}(k(x))\right) \cong k$ for all $x \in X$;

2. $\operatorname{Hom}_{Y}\left(\Phi_{\mathcal{E}}(k(x)), \Phi_{\mathcal{E}}(k(x))[i]\right) \cong 0$ for all $x \in X$ and $i \notin[0, \operatorname{dim} X]$;

3. $\operatorname{Hom}_{Y}\left(\Phi_{\mathcal{E}}(k(x)), \Phi_{\mathcal{E}}(k(y))[i]\right) \cong 0$ for all $x, y \in X$ such that $x \neq y$ and $i \in \mathbb{Z}$.

Vanishing results To apply the Bondal-Orlov criterion in the proof of theorem A we need the following generalisation of [14, parts (1), (2) and (3) of theorem 1.2]. For any point $z \in C$, we denote by $\mathcal{W}_{z}$ the restriction of $\mathcal{W}$ to $\{z\} \times C$. 
Theorem 3. Let $C$ be a smooth projective curve of genus $g \geq g_{0}$, and $\mathcal{L}$ a line bundle of degree 1 . Let $\mathcal{W}$ be the normalised Poincaré bundle on $C \times \mathrm{M}_{C}(r, \mathcal{L})$. Then

1. $\mathrm{H}^{0}\left(\mathrm{M}_{C}(r, \mathcal{L}), \mathcal{W}_{x} \otimes \mathcal{W}_{x}^{\vee}\right) \cong k$ for all $x \in C ;$

2. $\mathrm{H}^{i}\left(\mathrm{M}_{C}(r, \mathcal{L}), \mathcal{W}_{x} \otimes \mathcal{W}_{x}^{\vee}\right) \cong 0$ for all $x \in C$ and $i \geq 2$;

3. $\mathrm{H}^{i}\left(\mathrm{M}_{C}(r, \mathcal{L}), \mathcal{W}_{x} \otimes \mathcal{W}_{y}^{\vee}\right) \cong 0$ for all $x, y \in C$ such that $x \neq y$ and $i \geq 0$.

For the proof of the semiorthogonal decomposition of theorem B we need another set of vanishing results, which generalise [14, parts (4) and (5) of theorem 1.2], together with a new vanishing result.

Theorem 4. Let $C$ be a smooth projective curve of genus $g \geq g_{0}$, and $\mathcal{L}$ a line bundle of degree 1 . Let $\mathcal{W}$ be the normalised Poincaré bundle on $C \times \mathrm{M}_{C}(r, \mathcal{L})$. Then

1. $\mathrm{H}^{i}\left(\mathrm{M}_{C}(r, \mathcal{L}), \mathcal{W}_{x}^{\vee}\right)=0$ for all $x \in C$ and all $i \geq 0$.

2. $\mathrm{H}^{i}\left(\mathrm{M}_{C}(r, \mathcal{L}), \mathcal{W}_{x}^{\vee} \otimes \Theta^{\vee}\right)=0$ for all $x \in C$ and all $i \geq 0$.

2. $\mathrm{H}^{i}\left(\mathrm{M}_{C}(r, \mathcal{L}), \mathcal{W}_{x} \otimes \Theta^{\vee}\right)=0$ for all $i \geq 0$.

3. $\mathrm{H}^{i}\left(\mathrm{M}_{C}(r, \mathcal{L}), \mathcal{W}_{x}^{\vee} \otimes \mathcal{W}_{y} \otimes \Theta^{\vee}\right)=0$ for any $x, y \in C$ and all $i \geq 0$.

Remark 5. If $r=2$ then parts (1) and (2) are related via Serre duality, but in higher rank this is no longer the case. In arbitrary rank, (2) and (2') are related via Serre duality.

Structure of the paper We prove parts (1) and (2) of theorem 3 in section 3 . The proof of part (3) is more technical, and occupies sections 5 and 6 . The structure of the proof is as in [14], but we highlight the complications arising in the higher rank case, and why one only gets a proof in the case where $d=1$.

The proof of parts (1) and (2) (resp. (2')) of theorem 4 is done in section 7, where we prove a more general vanishing statement for exterior powers of $\mathcal{W}_{x}^{\vee}$, along the same lines as sections 5 and 6. Finally, the proof of part (3) occupies section 8 .

In section 9 we explain how theorems A and B follows from theorems 3 and 4, and give some concluding remarks.

Acknowledgements Both authors were supported by the Max Planck Institute for Mathematics in Bonn. They thank Patrick Brosnan and Sergey Galkin for interesting conversations.

\section{Preliminaries and notation}

Let $k$ be an algebraically closed field of characteristic 0 . Throughout this article we will take $C$ a smooth projective curve over $k$ of genus $g \geq 2$. Associated to a rank $r$ and degree $d$ such that $\operatorname{gcd}(r, d)=1$ there exists a smooth projective moduli space $\mathrm{M}_{C}(r, d)$ of dimension $r^{2}(g-1)+1$, with a determinant morphism to $\operatorname{Pic}^{d}(C)$. Fixing a line bundle $\mathcal{L}$ of degree $d$ on $C$ we can consider the fibre over the point $[\mathcal{L}]$ in $\operatorname{Pic}^{d}(C)$, which will be denoted $\mathrm{M}_{C}(r, \mathcal{L})$. 
Now $\mathrm{M}_{C}(r, \mathcal{L})$ has $\operatorname{Pic}\left(\mathrm{M}_{C}(r, \mathcal{L})\right) \cong \mathbb{Z} \Theta$ with $\Theta$ the ample generator of the Picard group, such that

(5) $\quad \omega_{\mathrm{M}_{C}(r, \mathcal{L})} \cong \Theta^{\otimes-2}$.

In particular, $\mathrm{M}_{C}(r, \mathcal{L})$ is a $\left(r^{2}-1\right)(g-1)$-dimensional Fano variety of index 2 . We will also use the appropriate analogues of these results when $\operatorname{gcd}(r, d) \geq 2$. For a proof one is referred to [8].

As $\mathrm{M}_{C}(r, \mathcal{L})$ is a fine moduli space, there exists a universal family $\mathcal{W}$ on $C \times \mathrm{M}_{C}(r, \mathcal{L})$, the Poincaré bundle. This universal family is unique if we normalise it as in $[18$, remark 2.9]: if we denote $\ell \geq 0$ the minimal integer such that $\ell d \equiv 1 \bmod r$ then we can assume

(6) $c_{1}\left(\mathcal{W}_{x}\right) \cong \Theta^{\otimes \ell}$,

where $\mathcal{W}_{x}=\mathcal{W}_{\{x\} \times \mathrm{M}_{C}(r, \mathcal{L})}$.

Remark 6. The dependence on $\ell$ is the reason why we have to restrict to $d \equiv 1 \bmod r$. Remark that by Serre duality and tensoring with $\mathcal{O}_{C}(x)$ we can always assume $d \in[0, \ldots, r / 2]$, as one can identify the moduli spaces for different $d$, so we will use $d=1$. We expect that for other residue classes the result still holds.

Using $\mathcal{W}$ and the projections

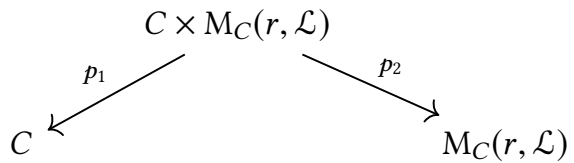

we can then construct the Fourier-Mukai functor

(8) $\Phi_{\mathcal{W}}=\mathrm{R} p_{2, *}\left(p_{1}^{*}(-) \otimes \mathcal{W}\right): \mathrm{D}^{\mathrm{b}}(C) \rightarrow \mathrm{D}^{\mathrm{b}}\left(\mathrm{M}_{C}(r, \mathcal{L})\right)$.

\section{Generalisation of a result by Narasimhan-Ramanan}

In this section we prove parts (1) and (2) of theorem 3. At this point it is not required that $d=1$, it is enough that $\operatorname{gcd}(r, d)=1$.

For (1) it suffices to observe that the result cited in [14] (i.e. [15, theorem 2(b)]) is already valid for all ranks. However for the proof of (2) [15, proposition 7.7] is used, which is only given for $g=2$. We will show that the expectation expressed in [15, remark 7.2] is correct, and that the vanishing of cohomology of $\operatorname{ad}_{x} \mathcal{W}$ (i.e. the restriction to $\{x\} \times \mathrm{M}_{C}(r, \mathcal{L})$ of the traceless endomorphisms of $\left.\mathcal{W}\right)$ is indeed valid in arbitrary rank, using some new information on Hodge numbers which was not available when op. cit. was published.

Proposition 7. Let $C$ be a smooth projective curve of genus $g \geq 2$, and $\mathcal{L}$ a line bundle of degree $d$ on $C$ such that $\operatorname{gcd}(r, d)=1$. Let $\mathcal{W}$ be the normalised Poincare bundle on $C \times \mathrm{M}_{C}(r, \mathcal{L})$, where $\operatorname{gcd}(r, d)=1$. For all $x \in C$ and $i \geq 2$ we have that

(9) $\quad \mathrm{H}^{i}\left(\mathrm{M}_{C}(r, \mathcal{L}), \operatorname{ad}_{x} \mathcal{W}\right)=0$ 
The proof of [15, proposition 7.7] can be generalised to arbitrary rank, provided one has control over the Hodge numbers $h^{1, i}\left(\mathrm{M}_{C}(r, \mathcal{L})\right)$. In order to do this, we will use the closed formula for the Hodge-Poincaré polynomial as obtained in del Baño [2, corollary 5.1]. Recall that the Hodge-Poincaré polynomial $\operatorname{HP}(X, x, y)$ of a smooth projective variety $X$ is given by $\sum_{p, q \geq 0} \mathrm{~h}^{p, q}(X) x^{p} y^{q}$.

The formula in loc. cit. gives the Hodge-Poincaré polynomial of $\mathrm{M}_{C}(r, d)$, and fixing the determinant changes the Hodge-Poincaré polynomial by removing a factor $(1+x)^{g}(1+y)^{g}$ arising from the Jacobian of $C^{1}$. Taking these observations into account, and denoting $\langle\alpha\rangle$ the decimal part of a real number $\alpha$, we have

$$
\begin{aligned}
& \mathrm{HP}\left(\mathrm{M}_{C}(r, \mathcal{L}), x, y\right)=\sum_{r_{1}+\ldots+r_{\ell}=r}(-1)^{\ell-1} \frac{\left((1+x)^{g}(1+y)^{g}\right)^{\ell-1}}{(1-x y)^{\ell-1}} \\
& \cdot \prod_{j=1}^{\ell} \prod_{i=1}^{r_{j}-1} \frac{\left(1+x^{i} y^{i+1}\right)^{g}\left(1+x^{i+1} y^{i}\right)^{g}}{\left(1-(x y)^{i}\right)\left(1-(x y)^{i+1}\right)} \\
& \cdot \prod_{j=1}^{\ell-1} \frac{1}{1-(x y)^{r_{j}+r_{j+1}}} \\
& \cdot(x y)^{\sum_{i<j} r_{i} r_{j}(g-1)+\sum_{i=1}^{\ell-1}\left(r_{i}+r_{i+1}\right)\left\langle-\left(r_{1}+\ldots+r_{i}\right) d / r\right\rangle}
\end{aligned}
$$

where we sum over all compositions of $r$.

From this we can read off the following dimensions.

Lemma 8. We have that

1. $\mathrm{h}^{0,1}\left(\mathrm{M}_{C}(r, \mathcal{L}),\right)=0$,

2. $\mathrm{h}^{1,1}\left(\mathrm{M}_{C}(r, \mathcal{L}),\right)=1$,

3. $\mathrm{h}^{2,1}\left(\mathrm{M}_{C}(r, \mathcal{L}),\right)=g$,

4. $\mathrm{h}^{i, 1}\left(\mathrm{M}_{C}(r, \mathcal{L}),\right)=0$ for all $i \geq 3$.

Proof. We observe that the only composition $r=r_{1}+\ldots+r_{\ell}$ contributing to the coefficient of $x^{i} y$ is the composition with $\ell=1$. Indeed, developing all the denominators as a power series in $x y$ and multiplying them, we see that factor on the fourth line of (10) is zero for $\ell=1$ and bounded below by 2 for $\ell \geq 2$. Hence for the equalities in the lemma, it suffices to understand the contribution with $\ell=1$.

In this case the only non-trivial factor in (10) is the second one. It now suffices to observe that there will not be a monomial $y$, that $x y$ appears with coefficient 1 by the factor $(1-x y)$ in the denominator for $i=1$, that $x^{2} y$ appears with coefficient $g$ by the factor $\left(1+x^{2} y\right)^{g}$ for $i=2$ and that there cannot be any monomials of the form $x^{i} y$ for $i \geq 3$.

Proof of part (2) of theorem 3. It suffices to combine proposition 7 with the defining short exact sequence

(11) $\quad 0 \rightarrow \operatorname{ad}_{x} \mathcal{W} \rightarrow \mathcal{E} \operatorname{nd}\left(\mathcal{W}_{x}\right) \cong \mathcal{W}_{x} \otimes \mathcal{W}_{x}^{\vee} \rightarrow \mathcal{O}_{\mathrm{M}_{C}(r, \mathcal{L})} \rightarrow 0$

\footnotetext{
${ }^{1}$ There is a minor typo in the second summation in the exponent of the last factor in [2, corollary 5.1]
} 
and the vanishing of the higher cohomology of the structure sheaf as $\mathrm{M}_{C}(r, \mathcal{L})$ is Fano.

Remark 9. One can actually prove more. Recall that the level of a Hodge structure is defined as the maximum value of $|p-q|$ as $(p, q)$ ranges over $\mathrm{h}^{p, q} \neq 0$. In [6] it was shown that for $\mathrm{M}_{C}(2, \mathcal{L})$ the level of $\mathrm{H}^{i}\left(\mathrm{M}_{C}(2, \mathcal{L}), \mathbb{Q}\right)$ was bounded above by $\lfloor i / 3\rfloor$. For $r \geq 3$ the same is true, which immediately implies lemma 8. For $i \gg 0$ this bound can even be improved, but we haven't found a nice closed formula for it.

\section{Determinant of cohomology}

One of the main ingredients in the proof of part (3) of theorem 3 is an explicit description of the determinant of cohomology. The following proposition is a generalisation of [14, proposition 3.1], taking the extra complication for arbitrary $r$ and $d$ such that $\operatorname{gcd}(r, d)=1$ into account.

Proposition 10. Let $C$ be a smooth projective curve of genus $g \geq 2$, and $\mathcal{L}$ a line bundle of degree $d$ on $C$ such that $\operatorname{gcd}(r, d)=1$. Let $\mathcal{W}$ be the normalised Poincaré bundle on $C \times \mathrm{M}_{C}(r, \mathcal{L})$, where $\operatorname{gcd}(r, d)=1$. Then there exists an isomorphism

(12) $\operatorname{det}\left(\mathrm{R} p_{2, *}(\mathcal{W})^{\vee}\right)^{\vee} \cong \mathcal{L}^{\otimes(1-\ell d) / r+\ell(1-g)}$

where $\ell \geq 0$ is minimal such that $\ell d \equiv 1 \bmod r$.

Proof. Consider the moduli space $\mathrm{M}_{C}\left(r, \mathcal{L}^{\vee}\right)$. The family of vector bundles $\mathcal{W}^{\vee}$ on $C \times \mathrm{M}_{C}(r, \mathcal{L})$ gives an isomorphism

(13) $\phi: \mathrm{M}_{C}(r, \mathcal{L}) \stackrel{\cong}{\rightarrow} \mathrm{M}_{C}\left(r, \mathcal{L}^{\vee}\right)$.

Let us denote the ample generators of $\operatorname{Pic}\left(\mathrm{M}_{C}(r, \mathcal{L})\right)\left(\operatorname{resp} . \operatorname{Pic}\left(\mathrm{M}_{C}\left(r, \mathcal{L}^{\vee}\right)\right)\right)$ by $\Theta\left(\operatorname{resp} . \Theta^{\prime}\right)$. By [14, proposition 2.1] we get that

(14) $\Theta \cong \phi^{*}\left(\Theta^{\prime}\right) \cong\left(\operatorname{det}\left(\mathbf{R} p_{2, *} \mathcal{W}^{\vee}\right)^{\vee}\right)^{\otimes r} \otimes \operatorname{det}\left(\mathcal{W}_{x}^{\vee}\right)^{\otimes-d+r(1-g)}$.

Because $\mathcal{W}$ is normalised we get $\operatorname{det} \mathcal{W}_{x} \cong \Theta^{\ell}$ as explained in section 2, where $\ell$ is the minimal non-negative integer such that $\ell d \equiv 1 \bmod r$. Hence we obtain

$$
\Theta \cong\left(\operatorname{det}\left(\mathbf{R} p_{2, *} \mathcal{W}^{\vee}\right)^{\vee}\right)^{\otimes r} \otimes \Theta^{\otimes \ell d+\ell r(g-1)} .
$$

This proves the proposition.

In appendix A we give an alternative proof of this result using the GrothendieckRiemann-Roch formula.

\section{Cohomology vanishing for $\mathcal{W}_{x} \otimes \mathcal{W}_{y}^{\vee}$ in high de- grees}

From this point on we will impose the condition that $d=1$. As mentioned before, we expect the result is still valid for any degree coprime to the rank, but leave this for future work. 
We will split the proof of the vanishing as stated in part (3) of theorem 3 into two parts: first we show it for $i \geq r^{2}$, and then we deal with the vanishing in low degrees in section 6. For both parts we use the Hecke correspondence, which we will recall now.

Hecke correspondence Let $x \in C$ be a closed point. If we restrict the Poincaré bundle $\mathcal{W}$ to $\{x\} \times \mathrm{M}_{C}(r, \mathcal{L})$, we can consider the projective bundle $\pi: \mathbb{P}\left(\mathcal{W}_{x}\right) \rightarrow \mathrm{M}_{C}(r, \mathcal{L})$. We will denote the projective bundle by $Q(\mathcal{W}, x)$.

A point $q$ on $Q(\mathcal{W}, x)$ corresponds to the vector bundle $\mathcal{W}_{\pi(q)}$ together with a morphism $\mathcal{W}_{\pi(q)} \rightarrow k(x)$. We can take the dual of the kernel, which is a vector bundle of rank $r$ and determinant $\mathcal{L}^{\vee} \otimes \mathcal{O}_{C}(x)$ of degree $1-d$. The variety $Q(\mathcal{W}, x)$ can be seen to parametrise a family of such bundles. However in general the family of vector bundles may not be semistable.

Consider the short exact sequence

$$
0 \rightarrow \varepsilon_{1} \rightarrow \mathcal{E} \rightarrow k(x) \rightarrow 0
$$

on a curve $C$, where $\mathcal{E}_{1}$ and $\mathcal{E}$ are vector bundles of rank $r$ and $\operatorname{deg}(\mathcal{E})=1$. Then we have the following:

Lemma 11. If $\mathcal{E}$ is stable, then $\mathcal{E}_{1}$ is semistable.

Proof. First observe that the slope $\mu\left(\mathcal{E}_{1}\right)=0$. Suppose that $\mathcal{E}$ is not semistable. Let $\mathcal{F}^{\prime}$ be a subbundle of $\mathcal{E}_{1}$ such that $\mu\left(\mathcal{F}^{\prime}\right)>\mu\left(\mathcal{E}_{1}\right)=0$. Now since $\mathcal{E}$ is stable, we have

(17) $0<\mu(F)<\mu(E)=\frac{1}{r}$.

But this is impossible since $0<r^{\prime}<r$ and $\operatorname{deg}\left(\mathcal{F}^{\prime}\right)>0$.

The above lemma shows that Hecke transforms for degree one bundles preserves semistability. Thus we get a diagram

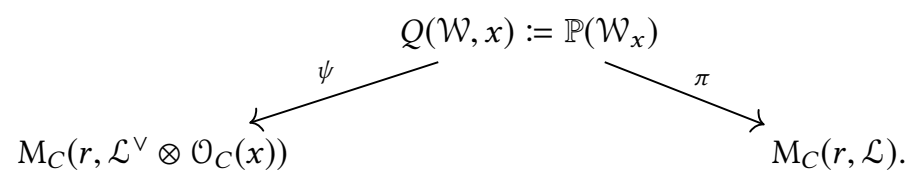

Remark 12. When $d \neq 1$ we don't have a well-defined morphism $\psi$ in (18), there is only a rational morphism. It is conceivable that using parabolic bundles it is possible to resolve the indeterminacy, and continue the proof in this way. We leave this for future work.

With the above considerations, the statement and proof of [14, proposition 3.3] generalises to the following.

Lemma 13. Let $\Theta^{\prime}$ be the ample generator of $\operatorname{Pic}\left(\mathrm{M}_{C}\left(r, \mathcal{L}^{\vee} \otimes \mathcal{O}_{C}(x)\right)\right.$. Then

$$
\psi^{*}\left(\Theta^{\prime}\right) \cong \mathcal{O}_{Q(\mathcal{W}, x)}(1)
$$

The following proposition is a generalisation of [14, proposition 3.2]. 
Proposition 14. Let $C$ be a smooth projective curve of genus $g \geq 2$, and $\mathcal{L}$ a line bundle of degree 1 on $C$. Let $\mathcal{W}$ be the normalised Poincaré bundle on $C \times \mathrm{M}_{C}(r, \mathcal{L})$. For all $x, y \in C$ such that $x \neq y$ the vector bundle $\mathcal{W}_{x} \otimes \mathcal{W}_{y}^{\vee} \otimes \omega_{\mathrm{M}_{C}(r, \mathcal{L})}^{\vee}$ is ample.

Proof. By lemma 13 we get that $\mathcal{O}_{Q(\mathcal{W}, x)}(1)$ is nef, hence $\mathcal{W}_{x}$ is nef. By [13, proposition 6.2.12(iv)] the exterior power $\bigwedge^{r-1} \mathcal{W}_{x}$ is again nef, and so $\wedge^{i} \mathcal{W}_{x} \otimes \Theta$ is ample. Using the ampleness of

$$
\begin{aligned}
\bigwedge^{r-1} \mathcal{W}_{x} \otimes \Theta & \cong \bigwedge^{r-1} \mathcal{W}_{x} \otimes \Theta^{\vee} \otimes \mathcal{L}^{\otimes 2} \\
& \cong \bigwedge^{r-1} \mathcal{W}_{x} \otimes \operatorname{det} \mathcal{W}_{x}^{\vee} \otimes \Theta^{\otimes 2} \\
& \cong \mathcal{W}_{x}^{\vee} \otimes \omega_{\mathrm{M}_{C}(r, \mathcal{L})}^{\vee}
\end{aligned}
$$

we can conclude that

$$
\begin{aligned}
\mathcal{W}_{x} \otimes \mathcal{W}_{y}^{\vee} \otimes \omega_{M_{C}(r, \mathcal{L})}^{\vee} & \cong \mathcal{W}_{x} \otimes \Theta \otimes \mathcal{W}_{y}^{\vee} \otimes \Theta \\
& \cong \mathcal{W}_{x} \otimes \Theta \otimes \bigwedge^{r-1} \mathcal{W}_{y}
\end{aligned}
$$

is again the tensor product of a nef bundle with an ample bundle, so is ample itself.

From this we get the vanishing of $\mathcal{W}_{x} \otimes \mathcal{W}_{y}^{\vee}$ in high degree.

Corollary 15. We have that for $i \geq r^{2}$.

$$
\mathrm{H}^{i}\left(\mathrm{M}_{C}(r, \mathcal{L}), \mathcal{W}_{x} \otimes \mathcal{W}_{y}^{\vee}\right)=0
$$

Proof. It suffices to apply Le Potier vanishing [13, theorem 7.3.5] to the ample vector bundle $\mathcal{W}_{x} \otimes \mathcal{W}_{y}^{\vee} \otimes \omega_{M_{C}(r, \mathcal{L})}^{\vee}$ of rank $r^{2}$.

\section{Cohomology vanishing for $\mathcal{W}_{x} \otimes \mathcal{W}_{y}^{\vee}$ in low degrees}

To finish the proof of part (3) theorem 3 we need to show vanishing in degrees $i \leq r^{2}-1$. For this we will use the morphism $\psi$ from (18).

Because $\operatorname{deg} \mathcal{L}^{\vee} \otimes \mathcal{O}_{C}(x)=0$ is not coprime with $r$, there is a dense open subset

$$
\mathrm{M}_{C}^{\mathrm{s}}\left(r, \mathcal{L}^{\vee} \otimes \mathcal{O}_{C}(x)\right) \subsetneq \mathrm{M}_{C}\left(r, \mathcal{L}^{\vee} \otimes \mathcal{O}_{C}(x)\right)
$$

of stable vector bundles, the complement being the strictly semistable locus.

Remark 16. If we take a stable bundle $\mathcal{V} \in \mathrm{M}_{C}\left(r, \mathcal{L}^{\vee} \otimes \mathcal{O}_{C}(x)\right)$, then by [16, remark 5.2(v) and lemma 5.6(i)] the Hecke transform of $\mathcal{V}$ is also stable.

Using remark 16, the proof of [14, proposition 3.4] also gives a proof of the following generalisation.

Proposition 17. The restriction of $\psi$ to $\mathrm{M}_{C}^{\mathrm{s}}\left(r, \mathcal{L}^{\vee} \otimes \mathcal{O}_{C}(x)\right)$ is a $\mathbb{P}^{r-1}$-fibration. Moreover, for every point $y \in C \backslash\{x\}$ the restriction of $\pi^{*}\left(\mathcal{W}_{y}\right)$ to a fibre of $\psi$ in the stable locus is isomorphic to $\mathcal{O}_{\mathbb{P}^{r-1}}(1)^{\oplus r}$.

For completeness' sake, we give the proof. 
Proof. Consider a point $m \in \mathrm{M}_{C}^{\mathrm{s}}\left(r, \mathcal{L}^{\vee} \otimes \mathcal{O}_{C}(x)\right)$, which corresponds to a stable vector bundle $\mathcal{V}$ on $C$ of rank $r$ and determinant $\mathcal{L}^{\vee} \otimes \mathcal{O}_{C}(x)$. We wish to show that

(24) $\left.\pi^{*}\left(\mathcal{W}_{y}\right)\right|_{\psi^{-1}(m)} \cong \mathcal{O}_{\mathbb{P}^{r-1}}(1)^{\oplus r}$.

Let us denote $V_{x}=\mathcal{V}_{x} \otimes k(x)$ the fibre of $\mathcal{V}$ at $x$. Then as in [15, §4] we obtain a family of vector bundles $K(\mathcal{V})$ on $C$ parametrised by $\mathbb{P}\left(V_{x}\right) \cong \mathbb{P}^{r-1}$, where $T=$ Spec $k$ is just a point. It should be mentioned that we will also use results from [16, §5], and it is important to highlight remark 5.7 of op. cit., which explains how the results in $\S 5$ of op. cit. are to be interpreted in the context of $[15, \S 4]$, in particular we have $x \in C$ fixed.

As $K(\mathcal{V})$ is a family of stable vector bundles of rank $r$ and determinant $\mathcal{L}$, the universal property of $\mathrm{M}_{C}(r, \mathcal{L})$ gives us a morphism

(25) $f: \mathbb{P}\left(V_{x}\right) \rightarrow \mathrm{M}_{C}(r, \mathcal{L})$

which is a closed immersion by [16, lemma 5.9], where in the setting of loc. cit. we restricted the morphism to the point $x \in C$. The same universal property gives us an isomorphism

(26) $\left(f \times \mathrm{id}_{C}\right)^{*}(\mathcal{W}) \cong K(\mathcal{V}) \otimes g^{*}(\xi)$

on $\mathbb{P}\left(V_{x}\right) \times C$, for some line bundle $\xi$ on $\mathbb{P}\left(V_{x}\right)$, where $g: \mathbb{P}\left(V_{x}\right) \times C \rightarrow \mathbb{P}\left(V_{x}\right)$ is the projection onto the first factor. We wish to determine the line bundle $\xi$ more explicitly, and we do this by computing the restriction $f^{*}(\Theta)$.

First, by [16, remark 5.7 and corollary 5.16] we get an isomorphism

(27) $f^{*}\left(\omega_{\mathrm{M}_{C}}(r, \mathcal{L})\right) \cong \omega_{\mathbb{P}\left(V_{x}\right)}^{\otimes 2}$,

and as $\mathrm{M}_{C}(r, \mathcal{L})$ is Fano of index 2 we get

(28) $f^{*}(\Theta) \cong \mathcal{O}_{\mathbb{P}\left(V_{x}\right)}(r)$.

On the other hand, using (26) we get

$$
\begin{aligned}
f^{*}(\Theta) & \cong f^{*}\left(\operatorname{det}\left(\mathcal{W}_{y}\right)\right) \\
& \cong \operatorname{det}\left(\left.K(\mathcal{V})\right|_{\mathbb{P}\left(V_{x}\right) \times\{y\}} \otimes \xi\right) .
\end{aligned}
$$

But as $\left.K(\mathcal{V})\right|_{\mathbb{P}\left(V_{x}\right) \times\{y\}} \cong \mathcal{O}_{\mathbb{P}\left(V_{x}\right)}^{\oplus r}$ by [15, remark 4.7], we get

(30) $f^{*}(\Theta) \cong \xi^{\otimes r}$.

Hence $\xi \cong \mathcal{O}_{\mathbb{P}\left(V_{x}\right)}(1)$, and (26) gives

(31) $f^{*}\left(\mathcal{W}_{y}\right) \cong \mathcal{O}_{\mathbb{P}\left(V_{x}\right)}(1)^{\oplus r}$.

Finally, similar to $[15, \S 5]$ there exists a commutative diagram

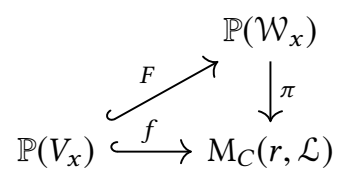

such that the image of $F$ is identified with $\psi^{-1}(m)$. But then (24) follows. 
We will need the following codimension estimate for the preimage under $\psi$ for the strictly semistable locus. The proof is similar to that in [19, §2.V.A], but we will now fix the determinant.

Lemma 18. Denoting $U:=\psi^{-1}\left(M_{C}^{\mathrm{s}}\left(r, \mathcal{L}^{\vee} \otimes \mathcal{O}_{C}(x)\right)\right.$ we have that

$$
\operatorname{codim}_{Q(\mathcal{W}, x)}(Q(\mathcal{W}, x) \backslash U) \geq 2(r-1)(g-1)-1
$$

Proof. Let $\mathcal{M}$ be a line bundle of degree $m$. Recall that polystable bundles in

(34) $\quad K:=\mathrm{M}_{C}(r, \mathcal{M}) \backslash \mathrm{M}_{C}^{\mathrm{s}}(r, \mathcal{M})$

are direct sums of stable bundles with the same slope. If we denote $a=\operatorname{gcd}(r, m) \geq 2$ and $r_{0}=r / a$ we get

$$
\begin{aligned}
\operatorname{dim} K & =\max _{c=1, \ldots,\lfloor a / 2\rfloor}\left(r_{0}^{2}\left(c^{2}+(a-c)^{2}(g-1)+2-g\right)\right. \\
& =\left(2 r_{0}^{2}+r^{2}-2 r r_{0}-1\right)(g-1)+1,
\end{aligned}
$$

where we can choose the determinant for one of the summands freely.

In the situation of (18) we have $d=1$, and hence $r_{0}=1$. By a generalisation of the proof of [19, lemma 5], it follows that the fibers of the map $\psi$ are at most $(r-1)$-dimensional. Thus we see that the dimension of $\psi^{-1}(K)$ is at most $\left(2+r^{2}-2 r-1\right)(g-1)+1+(r-1)$. Hence we get that

$$
\begin{aligned}
& \operatorname{codim}_{Q(\mathcal{W}, x)}(Q(\mathcal{W}, x) \backslash U) \\
& \quad=\left(r^{2}-1\right)(g-1)+r-1-\operatorname{dim} \psi^{-1}(K) \\
& \quad \geq 2(r-1)(g-1)-1 .
\end{aligned}
$$

Remark 19. If $r=2$, it is known that $\psi$ is surjective by [16, lemma 7.3] and the inequality in the statement of lemma 18 is an equality.

In corollary 15 we have shown the vanishing of $\mathrm{H}^{i}\left(\mathrm{M}_{C}(r, \mathcal{L}), \mathcal{W}_{x} \otimes \mathcal{W}_{y}^{\vee}\right)$ for $i \geq r^{2}$. We can now show vanishing for $i \leq r^{2}-1$.

Proof of part (3) of theorem 3. By the Leray spectral sequence for $\psi$ and (19) we get that

$$
\begin{aligned}
\mathrm{H}^{i}\left(\mathrm{M}_{C}(r, \mathcal{L}), \mathcal{W}_{x} \otimes \mathcal{W}_{y}^{\vee}\right) & \cong \mathrm{H}^{i}\left(Q(\mathcal{W}, x), \mathcal{O}_{Q(\mathcal{W}, x)}(1) \otimes \pi^{*}\left(\mathcal{W}_{y}^{\vee}\right)\right) \\
& \cong \mathrm{H}^{i}\left(Q(\mathcal{W}, x), \psi^{*}\left(\Theta^{\prime}\right) \otimes \pi^{*}\left(\mathcal{W}_{y}^{\vee}\right)\right)
\end{aligned}
$$

Let $g_{0}$ be the smallest integer such that

(38) $2 r g_{0}-2\left(r+g_{0}\right) \geq r^{2}-1$,

where we have used the codimension estimate from lemma 18. Then by [11, §III.3] we have an inclusion

(39) $\quad \mathrm{H}^{i}\left(Q(\mathcal{W}, x), \psi^{*}\left(\Theta^{\prime}\right) \otimes \pi^{*}\left(\mathcal{W}_{y}^{\vee}\right)\right) \hookrightarrow H^{i}\left(U, \psi^{*}\left(\Theta^{\prime}\right) \otimes \pi^{*}\left(\mathcal{W}_{y}^{\vee}\right)\right)$

for $i \leq r^{2}-1$. 
By proposition 17 we have that $\psi$ restricted to $U$ is a $\mathbb{P}^{r-1}$-fibration. Applying the projection formula

(40) $\quad \mathrm{R}^{j} \psi_{*}\left(\psi^{*}\left(\Theta^{\prime}\right) \otimes \pi^{*}\left(\mathcal{W}_{y}^{\vee}\right)\right) \cong \Theta \otimes \mathrm{R}^{j} \psi_{*}\left(\pi^{*}\left(\mathcal{W}_{y}^{\vee}\right)\right)$

and using that the fibers of $\pi^{*}\left(\mathcal{W}_{y}^{\vee}\right)$ restricted to $\psi^{-1}(p)$ for $p \in \mathrm{M}_{C}^{\mathrm{s}}\left(r, \mathcal{L}^{\vee} \otimes \mathcal{O}_{C}(x)\right)$ are isomorphic to $\mathcal{O}_{\mathbb{P} r-1}(-1)^{\oplus r}$ we are done.

\section{Cohomology vanishing for $\mathcal{W}_{x}^{\vee}$ and $\mathcal{W}_{x} \otimes \Theta^{\vee}$}

In this section we prove parts (1), (2) and (2') of theorem 4. The proof goes along the same lines as the proof of part (3) of theorem 3, and uses ingredients from sections 5 and 6.

To prove cohomology vanishing for $\mathcal{W}_{x}^{\vee}$ and $\mathcal{W}_{x} \otimes \Theta^{\vee}$ we prove the following more general vanishing statement for $\bigwedge^{j} \mathcal{W}_{x}^{\vee}$ with $j=1, \ldots, r-1$. Setting $j=1$ and $j=r-1$ then implies the result, as

$$
\bigwedge^{r-1} \mathcal{W}_{x}^{\vee} \cong \mathcal{W}_{x} \otimes \Theta^{\vee}
$$

because $\operatorname{det} \mathcal{W}_{x} \cong \Theta$.

Proposition 20. For any $i \geq 0$ and for $j=1, \ldots, r-1$ we have that

(42) $\mathrm{H}^{i}\left(\mathrm{M}_{C}(r, \mathcal{L}), \bigwedge^{j} \mathcal{W}_{x}^{\vee}\right)=0$

whenever $g \geq g_{j}$, where $g_{j}$ is the smallest positive integer satisfying the condition

(43) $2 r g_{j}-2\left(r+g_{j}\right) \geq\left(\begin{array}{l}r \\ j\end{array}\right)-1$.

Proof. The strategy of the proof is similar to the proof of part (3) of theorem 3. First we claim that $\bigwedge^{j} \mathcal{W}_{x}^{\vee} \otimes \omega_{\mathrm{M}_{C}(r, \mathcal{L})}$ is ample. This follows from the isomorphism

$$
\bigwedge^{j} \mathcal{W}_{x}^{\vee} \otimes \omega_{\mathrm{M}_{C}(r, \mathcal{L})} \cong \bigwedge^{r-j} \mathcal{W}_{x} \otimes \Theta
$$

and the fact that $\mathcal{W}_{x}$ is nef, using lemma 13, as in the proof of proposition 14

Thus by Le Potier vanishing [13, theorem 7.3.5] we get that

(45) $\mathrm{H}^{i}\left(\mathrm{M}_{C}(r, \mathcal{L}), \bigwedge^{j} \mathcal{W}_{x}^{\vee}\right)=0$

for $i \geq\left(\begin{array}{l}r \\ j\end{array}\right)$, as in section 5. Thus now we only have to prove vanishing when $i<\left(\begin{array}{l}r \\ j\end{array}\right)$. As in section 6 the Leray spectral sequence tells us

(46) $\mathrm{H}^{i}\left(\mathrm{M}_{C}(r, \mathcal{L}), \bigwedge^{j} \mathcal{W}_{x}^{\vee}\right) \cong \mathrm{H}^{i}\left(Q(\mathcal{W}, z), \bigwedge^{j} \pi^{*}\left(\mathcal{W}_{x}^{\vee}\right)\right.$,

where $z$ is any point on $C \backslash\{x\}$. 
Assuming that $g \geq g_{j}$ and using the codimension estimate of lemma 18 together with [11, §III.3], we obtain an inclusion

(47) $\mathrm{H}^{i}\left(Q(\mathcal{W}, z), \bigwedge^{j} \pi^{*}\left(\mathcal{W}_{x}^{\vee}\right) \subseteq \mathrm{H}^{i}\left(U, \bigwedge^{j} \pi^{*}\left(\mathcal{W}_{x}^{\vee}\right)\right.\right.$

for any $i<\left(\begin{array}{l}r \\ j\end{array}\right)$ and $U$ as in lemma 18 . Now by proposition 17 , we get that $\psi$ restricted to $U$ is a $\mathbb{P}^{r-1}$-fibration.

Moreover by proposition 17 , we see that the fiber of $\pi^{*}\left(\mathcal{W}_{x}^{\vee}\right)$ restricted to $\psi^{-1}(p)$ for $p \in \mathrm{M}_{C}^{\mathrm{s}}\left(r, \mathcal{L}^{\vee} \otimes \mathcal{O}_{C}(z)\right)$ is isomorphic to $\mathcal{O}_{\mathbb{P}^{r-1}}(-1)^{\oplus r}$. In particular, the fibers of $\bigwedge^{j} \pi^{*}\left(\mathcal{W}_{x}^{\vee}\right)$ restricted to $\psi^{-1}(p)$ are direct sums of line bundles of degree $-j$. This implies that $\mathrm{R}^{k} \psi_{*}\left(\bigwedge^{j} \pi^{*}\left(\mathcal{W}_{x}^{\vee}\right)\right)$ is zero, hence we are done.

\section{Cohomology vanishing for $\mathcal{W}_{x}^{\vee} \otimes \mathcal{W}_{y} \otimes \Theta^{\vee}$}

In this section we discuss the vanishing result of part (3) of theorem 4 required for the proof of theorem $\mathrm{B}$. We will recall the notion of $k$-ample vector bundles, as the role of Le Potier vanishing in the proof of theorem 3 will be replaced by Sommese vanishing.

Definition 21. Let $X$ be a projective variety. A line bundle $\mathcal{L}$ is said to be $k$-ample if

1. some power of $\mathcal{L}$ is globally generated, i.e. $\mathcal{L}$ is semi-ample;

2. the fibers of the morphism

(48) $\quad X \rightarrow \mathbb{P}\left(\mathrm{H}^{0}\left(X, \mathcal{L}^{\otimes r}\right)^{\vee}\right)$

have dimension at most $k$.

A vector bundle $\mathcal{E}$ on $X$ is said to be $k$-ample if the line bundle $\mathcal{O}_{\mathbb{P}(\mathcal{E})}(1)$ is $k$-ample.

The notion of 0 -ampleness agrees with ampleness and furthermore $k$-ampleness implies $(k+1)$-ampleness.

Proposition 22. Let $C$ be a smooth projective curve of genus $g \geq 2$ and $\mathcal{L}$ a line bundle of degree 1. Let $\mathcal{W}$ be the normalised Poincaré bundle on $C \times \mathrm{M}_{C}(r, \mathcal{L})$. Then $\mathcal{W}_{x}$ is $(r-1)$-ample.

Proof. By the generalisation of [19, lemma 5] we know that the fibres of the morphism $\psi$ in the Hecke correspondence of (18) are at most $(r-1)$-dimensional. Moreover, we have already (see 19) shown that

(49) $\mathcal{O}_{Q(\mathcal{W}, x)}(1) \cong \Theta^{\prime}$,

where $\Theta^{\prime}$ is the ample generator of the Picard group of $\mathrm{M}_{C}\left(r, \mathcal{L}^{\vee} \otimes \mathcal{O}_{C(x)}\right)$. Since $\Theta^{\prime}$ is (0-)ample, we get by [13, example 6.2.19(ii)]) that $\mathcal{O}_{Q(\mathcal{W}, x)}(1)$ is $(r-1)$-ample. In particular this implies that $\mathcal{W}_{x}$ is $(r-1)$-ample.

We will use this to prove part (3) of theorem 4.

Proof of part (3) of theorem 4. Let $x$ and $y$ be any two points of $C$, not necessarily distinct, and denote

(50) $\quad \mathcal{V}:=\mathcal{W}_{y} \otimes \mathcal{W}_{x}^{\vee} \otimes \Theta$ 
By proposition 22 we know that $\mathcal{W}_{x}$ and $\mathcal{W}_{y}$ are $(r-1)$-ample. Now by [12, theorem 3.5] we get that both $\bigwedge^{r-1} \mathcal{W}_{x}$ and $\mathcal{W}_{y} \otimes \bigwedge^{r-1} \mathcal{W}_{x}$ are $(r-1)$-ample. By our choice of normalisation of $\mathcal{W}$ there is a natural isomorphism

$$
\mathcal{V} \cong \mathcal{W}_{y} \otimes \mathcal{W}_{x}^{\vee} \otimes \Theta \cong \mathcal{W}_{y} \otimes \bigwedge^{r-1} \mathcal{W}_{x}
$$

Thus, we can conclude that the vector bundle $\mathcal{V}$ is $(r-1)$-ample. It follows by Sommese vanishing [20, proposition 1.14], that

$$
\mathrm{H}^{i}\left(\mathrm{M}_{C}(r, \mathcal{L}), \mathcal{W}_{y} \otimes \mathcal{W}_{x}^{\vee} \otimes \Theta^{\vee}\right)=0
$$

for all $i \geq r^{2}+(r-1)$.

Since $\omega_{\mathrm{M}_{C}(r, \mathcal{L})}^{\vee}$ is isomorphic to $\Theta^{\otimes 2}$ we get by Serre duality that

$$
\mathrm{H}^{i}\left(\mathrm{M}_{C}(r, \mathcal{L}), \mathcal{W}_{y} \otimes \mathcal{W}_{x}^{\vee} \otimes \Theta^{\vee}\right)^{\vee} \cong \mathrm{H}^{\left(r^{2}-1\right)(g-1)-i}\left(\mathrm{M}_{C}(r, \mathcal{L}), \mathcal{W}_{x} \otimes \mathcal{W}_{y}^{\vee} \otimes \Theta^{\vee}\right)
$$

The same argument shows that

$$
\mathrm{H}^{\left(r^{2}-1\right)(g-1)-i}\left(\mathrm{M}_{C}(r, \mathcal{L}), \mathcal{W}_{x} \otimes \mathcal{W}_{y}^{\vee} \otimes \Theta^{\vee}\right)=0
$$

when $\left(r^{2}-1\right)(g-1)-i \geq r^{2}+(r-1)$.

Combining the above with (52), we get that if $\left(r^{2}-1\right)(g-1) \geq 2\left(r^{2}+r-1\right)$, then the cohomology $\mathrm{H}^{i}\left(\mathrm{M}_{C}(r, \mathcal{L}), \mathcal{V}\right)$ vanishes for all $i$. But this is satisfied for all $g \geq 4$.

\section{Concluding remarks}

The proofs of theorems A and B We can now explain how the vanishing theorems imply the fully faithfulness of the Fourier-Mukai functor, and how they give the semiorthogonal decomposition of (4).

Proof of theorem A. The Bondal-Orlov criterion from proposition 2 can be applied using theorem 3 .

For the proof of theorem $B$ we need to use the following lemma.

Lemma 23. Let $X$ be a smooth projective variety. Let $F: \mathcal{A} \hookrightarrow \mathrm{D}^{\mathrm{b}}(X)$ and $G: \mathcal{B} \hookrightarrow \mathrm{D}^{\mathrm{b}}(X)$ be admissible embeddings. To check that $\mathcal{A}$ is in the right orthogonal to $\mathcal{B}$, it suffices to check this for spanning classes for $\mathcal{A}$ and $\mathcal{B}$, i.e. whether

(55) $\operatorname{Hom}_{\mathrm{D}^{\mathrm{b}}(X)}(G(T), F(S))=0$

for all objects $S$ in a spanning class for $\mathcal{A}$ and all objects $T$ in a spanning class for $\mathcal{B}$.

For sake of notational simplicity a spanning class will be closed under shifts.

Proof. We need to check that

(56) $\operatorname{Hom}_{\mathrm{D}^{\mathrm{b}}(X)}(G(B), F(A))=0$ 
for all $A \in \mathcal{A}$ and $B \in \mathcal{B}$. Applying the adjunction $G \dashv G^{\mathrm{R}}$ this is equivalent to $G^{\mathrm{R}} \circ F(A)$ being isomorphic to 0 for all $A$. This in turn is implied by

(57) $\operatorname{Hom}_{\mathcal{B}}\left(T, G^{\mathrm{R}} \circ F(A)\right)=0$,

where $T$ runs over a spanning class for $\mathcal{B}$. Now applying $G \dashv G^{\mathrm{R}}$ and $F^{\mathrm{L}} \dashv F$, this is equivalent to

(58) $\operatorname{Hom}_{\mathcal{A}}\left(F^{\mathrm{L}} \circ G(T), A\right)=0$,

which is equivalent to $F^{\mathrm{L}} \circ G(T)$ being isomorphic to zero for all $T$. This in turn is implied by

(59) $\operatorname{Hom}_{\mathcal{A}}\left(F^{\mathrm{L}} \circ G(T), S\right)=0$,

where $S$ runs over a spanning class for $\mathcal{A}$.

Proof of theorem B. The orthogonality criterion from lemma 23 can be applied to the images of $\mathrm{D}^{\mathrm{b}}(C)$ under $\Phi_{\mathcal{W}}$ and $\Phi_{\mathcal{W}} \otimes \Theta^{\vee}$ by using the spanning class given by the skyscrapers, and using part (3) of theorem 4.

The other orthogonality checks (there are 5 more) follow from Kodaira vanishing, and parts (1) and (2) of theorem 4.

On theorem B for rank 2 and genus 2 It is expected that in theorem A the condition on the genus is not essential, and combining [14, remark 5] with [9, theorem 1.1] we know that the functor $\Phi_{\mathcal{W}}$ is fully faithful for $r=2$ and all $g \geq 2$, so a posteriori we can conclude the vanishing results in theorem 3 .

But when $r=2$ and $g=2$ it is shown in [5, theorem 2.9] that (3) is the whole semiorthogonal decomposition, i.e.

(60) $\mathrm{D}^{\mathrm{b}}\left(\mathrm{M}_{C}(2, \mathcal{L})\right)=\left\langle\Theta^{\vee}, \mathcal{O}_{\mathrm{M}_{C}(2, \mathcal{L})}, \Phi_{\mathcal{W}}\left(\mathbf{D}^{\mathrm{b}}(C)\right)\right\rangle$.

In particular, theorem B cannot hold for $r=2$ and $g=2$, so we cannot have the vanishing result in part 3 of theorem 4 . We leave it to the interested reader to compute directly on the intersection $Q_{1} \cap Q_{2}$ of 2 smooth quadrics in $\mathbb{P}^{5}$ that the sheaf cohomology of the tensor product of the restriction of (dual) spinor bundles twisted by $\mathcal{O}_{Q_{1} \cap Q_{2}}(-1)$ is non-zero.

On the other hand, for all other combinations of rank and genus (and degree) it is expected that theorem $\mathrm{B}$ holds.

Generalised Picard bundles The fully faithfulness result from theorem A allows us to reprove known results on the inversion of generalised Picard bundles, and their deformation theory, originally proven in [1,4]. This is remarked upon in [14, remark 1] in the case when $r=2$. We will now give some details in the more general case here.

Definition 24. Let $\mathcal{E}$ be a semistable vector bundle of rank $n$ and degree $e$, such that

(61) $r e+n>r n(2 g-2)$.

Then the Fourier-Mukai transform $\Phi_{\mathcal{W}}(\mathcal{E})$ is again a vector bundle, of rank $r e+n+r n(1-g)$, called a generalised Picard bundle. 
The first result that follows from theorem $\mathrm{A}$ is [4, theorem 19]. Recall that the kernel $\mathcal{W}^{\mathrm{R}}$ for the right adjoint of $\Phi_{\mathcal{W}}$ is given by $\mathcal{W}^{\vee} \otimes p_{1}^{*}\left(\omega_{C}\right)[1]$. By fully faithfulness we have a natural equivalence $\Phi_{\mathcal{W}^{\mathrm{R}}} \circ \Phi_{\mathcal{W}} \cong \mathrm{id}_{\left.\mathrm{D}^{\mathrm{b}}(C)\right)}$.

Proposition 25 (Inversion formula). Let $\mathcal{E}$ be a vector bundle as in definition 24 . Then there exists an isomorphism

(62) $\mathcal{E} \cong \mathrm{R}^{1} p_{1, *}\left(p_{2}^{*}\left(p_{2, *}\left(p_{1}^{*}(\mathcal{E}) \otimes \mathcal{W}\right) \otimes \mathcal{W}^{\vee} \otimes p_{1}^{*}\left(\omega_{C}\right)\right)\right.$.

Similarly we can describe the deformation theory of generalised Picard bundles as in [4, theorem 22]. As the infinitesimal deformation theory of any sheaf $\mathcal{E}$ on a smooth projective variety $X$ is described by $\mathrm{H}^{i}(X, \mathcal{E} \operatorname{nd}(\mathcal{E}))$ for $i=0,1,2$, the fully faithfulness of $\Phi_{\mathcal{W}}$ gives the isomorphism in the next theorem.

Proposition 26. Let $\mathcal{E}$ be a vector bundle as in definition 24. Then there exists an isomorphism

(63) $\operatorname{Ext}_{C}^{i}(\mathcal{E}, \mathcal{E}) \cong \operatorname{Ext}_{\mathrm{M}_{C}(r, \mathcal{L})}^{i}\left(\Phi_{\mathcal{W}}(\mathcal{E}), \Phi_{\mathcal{W}}(\mathcal{E})\right)$

Proof. One uses that

(64) $\mathrm{H}^{i}(C, \mathcal{E} \operatorname{nd}(\mathcal{E})) \cong \operatorname{Ext}_{C}^{i}(\mathcal{E}, \mathcal{E})$

and

(65) $\mathrm{H}^{i}\left(\mathrm{M}_{C}(r, \mathcal{L}), \mathcal{E} \operatorname{nd}\left(\Phi_{\mathcal{W}}(\mathcal{E})\right)\right) \cong \operatorname{Ext}_{\mathrm{M}_{C}(r, \mathcal{L})}^{i}\left(\Phi_{\mathcal{W}}(\mathcal{E}), \Phi_{\mathcal{W}}(\mathcal{E})\right)$,

and the left-hand sides are isomorphic by fully faithfulness.

So the (infinitesimal) deformation theory of a generalised Picard bundle agrees with that of the original bundle. In particular, if $\mathcal{E}$ is simple, then by Riemann-Roch the deformation space has dimension $n^{2}(g-1)+1$.

\section{A An alternative proof of proposition 10}

Because $\mathrm{M}_{C}(r, \mathcal{L})$ is Fano, we have an isomorphism $\operatorname{Pic}\left(C \times \mathrm{M}_{C}(r, \mathcal{L})\right) \cong \operatorname{Pic} C \oplus \mathbb{Z}$. Using this isomorphism we have

(66) $\mathrm{c}_{1}(\mathcal{W})=\mathrm{c}_{1}\left(\mathcal{W}_{x}\right)+d$

For a vector bundle $\mathcal{E}$ we will use the following shorthand

(67) $\quad N_{2}(\varepsilon):=\mathrm{c}_{1}(\varepsilon)^{2}-2 \mathrm{c}_{2}(\mathcal{E})$.

We start with a preliminary lemma.

Lemma 27. We have an equality

(68) $r \cdot p_{2, *}\left(N_{2}(\mathcal{W})\right)=-2[\Theta]+2 d c_{1}\left(\mathcal{W}_{x}\right)$. 
Proof. By [18, theorem 1] we have that

$$
\begin{aligned}
-2[\Theta] & =\left[\omega_{\mathrm{M}_{C}(r, \mathcal{L})}\right] \\
& =\mathrm{c}_{1}\left(\operatorname{det} \mathbf{R} p_{2, *} \operatorname{ad} \mathcal{W}\right) .
\end{aligned}
$$

Using Grothendieck-Riemann-Roch we can further rewrite this to

$$
\begin{aligned}
\mathbf{c}_{1}\left(\operatorname{det} \mathbf{R} p_{2, *} \operatorname{ad} \mathcal{W}\right) & =\operatorname{ch}\left(p_{2, !} \operatorname{ad} \mathcal{W}\right)_{\operatorname{deg}=1} \\
& =p_{2, *}\left(\left(\operatorname{ch}(\operatorname{ad} \mathcal{W}) \otimes p_{1}^{*} \operatorname{td}\right)_{\operatorname{deg}=2}\right) \\
& =p_{2, *}\left(\left(\operatorname{ch}(\mathcal{E n d}(\mathcal{W})) \otimes p_{1}^{*} \operatorname{td}\right)_{\operatorname{deg}=2}\right) .
\end{aligned}
$$

Restricting ourselves to the terms that contribute to the part in degree 2 we obtain

$$
\begin{aligned}
p_{2, *} & \left(\left(\operatorname{ch}(\mathcal{E} \operatorname{nd}(\mathcal{W})) \otimes p_{1}^{*} \operatorname{td}_{C}\right)_{\mathrm{deg}=2}\right) \\
& =p_{2, *}\left(\left(\left(r^{2}+\frac{1}{2} N_{2}\left(\mathcal{W} \otimes \mathcal{W}^{\vee}\right)\right)\left(1+\frac{1}{2} p_{1}^{*} c_{1}\left(\mathrm{~T}_{X}\right)\right)\right)_{\mathrm{deg}=2}\right) \\
& =p_{2, *}\left(\frac{1}{2} N_{2}\left(\mathcal{W} \otimes \mathcal{W}^{\vee}\right)\right) \\
& =p_{2, *}\left(\frac{r}{2}\left(N_{2}(\mathcal{W})+N_{2}\left(\mathcal{W}^{\vee}\right)\right)+\mathrm{c}_{1}(\mathcal{W}) \mathrm{c}_{1}\left(\mathcal{W}^{\vee}\right)\right) \\
& =p_{2, *}\left(r N_{2}(\mathcal{W})-\mathrm{c}_{1}(\mathcal{W})^{2}\right) .
\end{aligned}
$$

We will now apply the normalisation for the Poincaré bundle $\mathcal{W}$.

Proof of proposition 10. By Grothendieck-Riemann-Roch we get

$$
\begin{aligned}
\mathrm{c}_{1}\left(\operatorname{det} \mathrm{R} p_{2, *} \mathcal{W}^{\vee}\right) & =\operatorname{ch}\left(p_{2, !} \mathcal{W}^{\vee}\right)_{\operatorname{deg}=1} \\
& =p_{2, *}\left(\operatorname{ch}\left(\mathcal{W}^{\vee}\right) p_{1}^{*} \operatorname{td}{ }_{C}\right) \\
& =p_{2, *}\left(\left(r-\mathrm{c}_{1}(\mathcal{W})+\frac{1}{2} N_{2}(\mathcal{W})\right)\left(1+\frac{1}{2} p_{1}^{*} c_{1}\left(\mathrm{~T}_{C}\right)\right)_{\operatorname{deg}=2}\right) \\
& =p_{2, *}\left(\frac{1}{2} \mathrm{c}_{1}(\mathcal{W}) p_{1}^{*} \mathrm{c}_{1}\left(\mathrm{~K}_{C}\right)\right)+\frac{1}{2} p_{2, *} N_{2}(\mathcal{W}) \\
& =(g-1) \mathrm{c}_{1}\left(\mathcal{W}_{x}\right)+\frac{1}{r}\left(-[\Theta]+d \mathrm{c}_{1}\left(\mathcal{W}_{x}\right)\right)
\end{aligned}
$$

where in the last step we used lemma 27. Now proposition 10 follows from (6).

\section{References}

[1] Vikraman Balaji and Periyapatna Vishwanath. "Deformations of Picard sheaves and moduli of pairs". In: Duke Mathematical fournal 76.3 (1994), pp. 773-792.

[2] Sebastian del Baño. "On the Chow motive of some moduli spaces". In: Journal für die Reine und Angewandte Mathematik 532 (2001), pp. 105-132.

[3] Pieter Belmans, Sergey Galkin, and Swarnava Mukhopadhyay. A semiorthogonal decomposition for moduli of vector bundles of rank 2. in progress. 
[4] Indranil Biswas, Leticia Brambila-Paz, and Peter Newstead. "Deformations of the generalised Picard bundle". In: Topology 45.2 (2006), pp. 403-419.

[5] Alexey Bondal and Dmitri Orlov. Semiorthogonal decomposition for algebraic varieties. 1995. arXiv: alg-geom/9506012 [math. AG].

[6] Sebastian Del Baño. "On the motive of moduli spaces of rank two vector bundles over a curve”. In: Compositio Mathematica 131.1 (2002), pp. 1-30.

[7] Usha Desale and Sundararaman Ramanan. "Classification of vector bundles of rank 2 on hyperelliptic curves". In: Inventiones Mathematicae 38.2 (1976/77), pp. 161-185.

[8] Jean-Marc Drézet and Mudumbai Narasimhan. "Groupe de Picard des variétés de modules de fibrés semi-stables sur les courbes algébriques”. In: Inventiones Mathematicae 97.1 (1989), pp. 53-94.

[9] Anton Fonarev and Alexander Kuznetsov. "Derived categories of curves as components of Fano manifolds". In: Journal of the London Mathematical Society. Second Series 97.1 (2018), pp. 24-46.

[10] Sergey Galkin, Vasily Golyshev, and Hiroshi Iritani. "Gamma classes and quantum cohomology of Fano manifolds: gamma conjectures”. In: Duke Mathematical fournal 165.11 (2016), pp. 2005-2077.

[11] Alexander Grothendieck. Cohomologie locale des faisceaux cohérents et théorèmes de Lefschetz locaux et globaux (SGA 2). North-Holland, 1968, pp. vii+287.

[12] Fatima Laytimi and Werner Nahm. Semiample and $k$-ample vector bundles. arXiv: 1607.07193v1 [math. AG].

[13] Robert Lazarsfeld. Positivity in algebraic geometry. II. Vol. 49. Ergebnisse der Mathematik und ihrer Grenzgebiete. 3. Folge. Springer-Verlag, 2004, pp. xviii+385. ISBN: 3-540-22534-X.

[14] Mudumbai Narasimhan. "Derived categories of moduli spaces of vector bundles on curves". In: Journal of Geometry and Physics 122 (2017), pp. 53-58.

[15] Mudumbai Narasimhan and Sundararaman Ramanan. "Deformations of the moduli space of vector bundles over an algebraic curve". In: Annals of Mathematics 101 (1975).

[16] Mudumbai Narasimhan and Sundararaman Ramanan. "Geometry of Hecke cycles. I". In: C. P. Ramanujam-a tribute. Vol. 8. Tata Inst. Fund. Res. Studies in Math. Springer, 1978, pp. 291-345.

[17] Shinnosuke Okawa. "Semi-orthogonal decomposability of the derived category of a curve". In: Advances in Mathematics 228.5 (2011), pp. 2869-2873.

[18] Sundararaman Ramanan. "The moduli spaces of vector bundles over an algebraic curve". In: Mathematische Annalen 200 (1973), pp. 69-84.

[19] Conjeeveram Seshadri. Fibrés vectoriels sur les courbes algébriques. Vol. 96. Astérisque. Notes written by J.-M. Drézet from a course at the École Normale Supérieure, June 1980. Société Mathématique de France, Paris, 1982, p. 209.

[20] Andrew John Sommese. "Submanifolds of abelian varieties". In: Mathematische Annalen 233.3 (1978), pp. 229-256. 